\title{
COMPARACIÓN DE MANEJOS PRATENSES DEL CENTRO-SUR DE CHILE UTILIZANDO VALORES BIOINDICADORES DE ELLENBERG
}

\section{COMPARISON OF PRAIRIE MANAGEMENTS IN SOUTH-CENTRAL CHILE WITH THE BIOINDICATORS VALUES OF ELLENBERG}

\author{
Cristina San Martín, Johana Villagra \& Carla Novoa \\ Instituto de Botánica, Universidad Austral de Chile, Casilla 567, Valdivia, Chile. \\ csanmart@uach.cl
}

\begin{abstract}
RESUMEN
Se compararon dos manejos pratenses: pastoreo con vacunos y pastoreo con ovejas, en un suelo volcánico, del centrosur de Chile, con los valores bioindicadores de Ellenberg establecidos para malezas europeas, que forman esas praderas. Se comprobó que el pastoreo con ovinos degrada el suelo y la cubierta vegetal mucho más que el pastoreo con bovinos, lo que se corresponde con las diferencias vegetacionales y edáficas establecidas por un estudio anterior mediante análisis edáficos y fitosociológicos. Con estos últimos se determinó que las diferencias entre ambos tipos de pastoreo establecieron dos asociaciones pratenses diferentes: Hyperico-Agrostietum capillariae (pastoreo de vacunos) y AiroAgrostietum capillariae (pastoreo de ovejas). Se confirmó que muy importante en el manejo pastoril es la degradación física del suelo, especialmente por compactación, que altera la cantidad, distribución por tamaño y orientación de los poros, cambiando la conductividad hidráulica y la proporción aire-agua-suelo, determinante en el arraigamiento de las hierbas pratenses. En ambos manejos es posible diferenciar distintos estadios de degradación, los que terminan por permitir el asentamiento de asociaciones arbustivas secundarias, tales como Aristotelio-Rubetum constrictae (matorral de zarzamora) en la pradera de vacuno. La degradación de la pradera de ovinos concluye en un matorral de rosa mosqueta no estudiado. Los valores indicadores biológicos por especie, muestran que los microclimáticos (luz y temperatura) tienden a concentrarse más en pocos tramos de la escala, mientras que los edáficos (reacción, nitrógeno y humedad del suelo) se dispersan en todo el espectro de valores indicadores. Las especies indiferentes a los bioindicadores aumentan con condiciones favorables, pero disminuyen en condiciones extremas. Por último, se confirma la utilidad de aplicar los valores bioindicadores de Ellenberg en comunidades vegetales pratenses chilenas dominadas por malezas de origen europeo, los cuales pueden captar incluso pequeñas diferencias en el manejo, sin necesidad de grandes inversiones de tiempo y dinero.
\end{abstract}

PALABRAS CLAVE: Fitoindicadores, praderas antropogénicas, manejo del suelo, pastoreo.

\section{ABSTRACT}

Ellenberg bioindicators values for European weeds were compared in two prairie managements with cattle and sheep on a volcanic soil of South-Central Chile. It was found that sheep grazing degrades the soil and vegetation cover much more that cattle grazing. These results corresponds to vegetation and soil differences found earlier in a plant sociological study, which found that the differences between both types of grazing established two different plant associations: Hyperico-Agrostietum capillariae (cattle grazing) and Airo-Agrostietum capillariae (sheep grazing). It was confirmed that with different kinds of management is very important the soil physical degradation, specially compactnees, that alters the quantity, size, distribution and orientation of the pores, changing the hydraulic conductivity and the air-water-soil ratio, determining the rooting of grasses. In both prairie is possible to distinguish different level of degradation, which ultimately allow the settlement of secondary shrub associations, such as Aristotelio-Rubetum constrictae (zarzamora scrub) in the cattle pasture. The degradation of the sheep prairie concluded in a not studied Hips Rose bush. The values of biological indicators plant species, show that those microclimate conditions (light and temperature) tend to focus more on small sections of the scale, while those edaphic (reaction, nitrogen and soil moisture) are dispersed throughout the spectrum of indicators values. The indifferent species to the bioindicators increased with favourable conditions but decreased in extreme conditions. Finally it confirms the usefulness of applying the values of Ellenberg bioindicators in the anthropogenic Chilean prairies, plant communities dominated by weeds of European origin, which can capture even small differences in handling, without requiring large investiment of time and money.

KEYwORDS: Plant indicators, anthropogenic prairie, soil management, grazing. 
Bioindicadores pratenses de Ellenberg: SAN Martín, C. ET AL.

\section{INTRODUCCION}

Los manejos agrícolas causan alteraciones en los suelos y las comunidades vegetales (Ellies 1995). Estas alteraciones son de orden químico y físico, siendo importante la pérdida de nutrientes y la alteración de la estructura del suelo en los horizontes superficiales, por el contrario, los manejos pecuarios tienden a mejorar el balance de nutrientes, pero crean alteraciones físicas en los horizontes edáficos profundos (Ramírez et al. 2005). La consolidación del suelo es una de ellas, la que al compactar los horizontes edáficos, cambia la relación entre los poros y la dirección de los mismos, alterando la relación aire-agua-suelo (Seguel et al. 2002a, 2002b).

Ramírez et al. (2005) investigaron dos manejos pecuarios, uno con bovinos y otro con ovinos en la zona de Chaquián (La Unión, Chile). Los resultados mostraron cambios en la estructura edáfica, pero también en la composición florística, a tal extremo que ambos presentaron dos praderas distintas, pertenecientes a las asociaciones vegetales: Hyperico-Agrostietum capillariae (Oberdorfer 1960) para la de vacunos y Airo-Agrostietum capillariae (Ramírez et al. 2003a) para la pastoreada con ovejas. Ambas praderas están dominadas por malezas de origen europeo, introducidas en tiempos históricos al país. Ellas han colonizado los biótopos ruderales creados por la intervención humana, adecuándose a sus cambios. Estas malezas reflejarán entonces, las condiciones físicas del biótopo y por lo tanto, podrían ser usadas como bioindicadores para hacer un diagnóstico del estado de éste. Ellenberg (1979) estableció una escala de indicadores biológicos para especies vegetales europeas con 9 tramos, considerando los factores: luz y temperatura del hábitat y reacción (acidez), nitrógeno y humedad del suelo, en la cual se encuentran prácticamente todas las especies constituyentes de las praderas permanentes del centro-sur de Chile (Oberdorfer 1960, Golf 1973).

Los valores indicadores biológicos establecidos por Ellenberg (1979) han sido evaluados repetidamente en distintos ambientes europeos (Diekmann 1995, Ertsen et al. 1998, Schaffers \& Sykora 2000, Godefroid \& Dana 2007), donde se obtienen correlaciones bastante aceptables de los factores ambientales y edáficos (Landolt 1977). También se ha demostrado su eficacia en la evaluación de biótopos terrestres y acuáticos en la región valdiviana (Chile) con praderas permanentes con alta antropización (Ramírez et al. 2000, 1995, 1992, San Martín et al. 2003).

La presente investigación hace una evaluación de dos praderas establecidas en Chaquián con diferentes manejos pecuarios, usando los valores indicadores biológicos de Ellenberg (1979) y de Ellenberg et al. (1992), para probar su utilidad en estos manejos pratenses en un suelo de ceniza volcánica antiguo del tipo rojo-arcilloso.

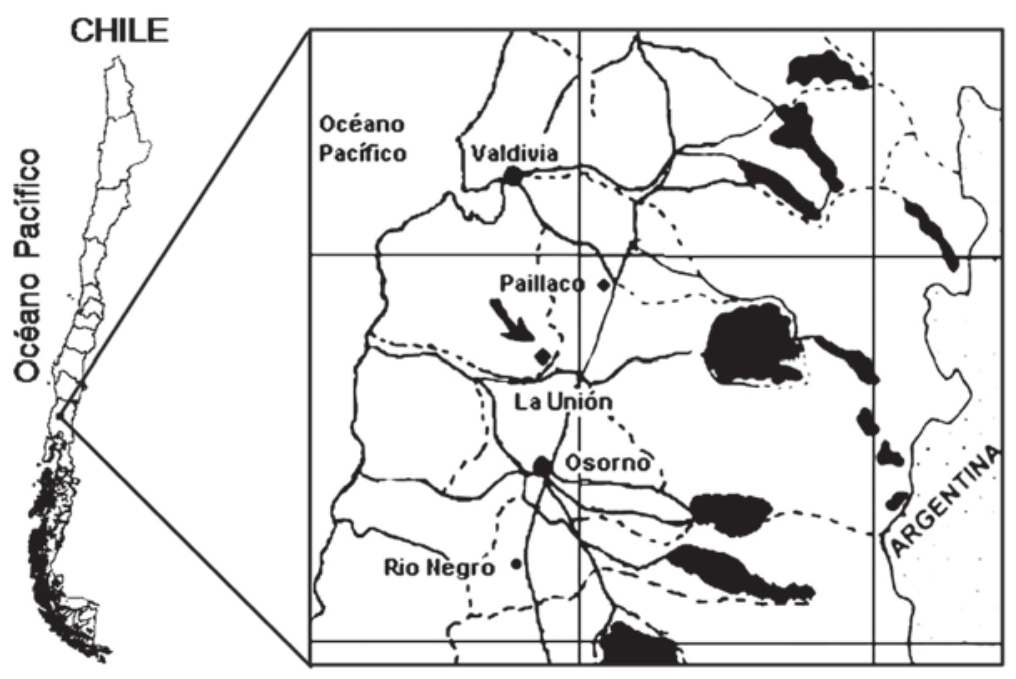

Figura 1. Area de estudio en el centro-sur de Chile. La flecha señala el lugar de trabajo.

Figure 1. Study area in South-Central Chile. The arrow indicates the study site. 
Gayana Bot. 66(2), 2009

\section{MATERIALES Y METODOS}

Area de estudio

El área de estudio (Fig. 1) se ubica $5 \mathrm{~km}$ al noroeste de la ciudad de La Unión (Región de Los Ríos, Chile) en el sector Chaquián ( $\left.40^{\circ} 13^{\prime} \mathrm{S}-73^{\circ} 08^{\prime} \mathrm{W}\right)$, en la base de la Cordillera de la Costa.

El clima del lugar de trabajo es templado húmedo con tendencia mediterránea (Di Castri \& Hajek 1976). Amigo \& Ramírez (1998) lo definen como un bioclima meso-temperado, el diagrama ombrotérmico de la Figura 2 resume las características de este clima (Novoa \& Villaseca 1989). La precipitación promedio anual llega a $1267 \mathrm{~mm}$ y la temperatura presenta un promedio anual mensual de $11,6^{\circ} \mathrm{C}$. Estos valores permiten clasificarlos como $\mathrm{Cfb}$ según Köppen y Geiger (Scherhag 1969).

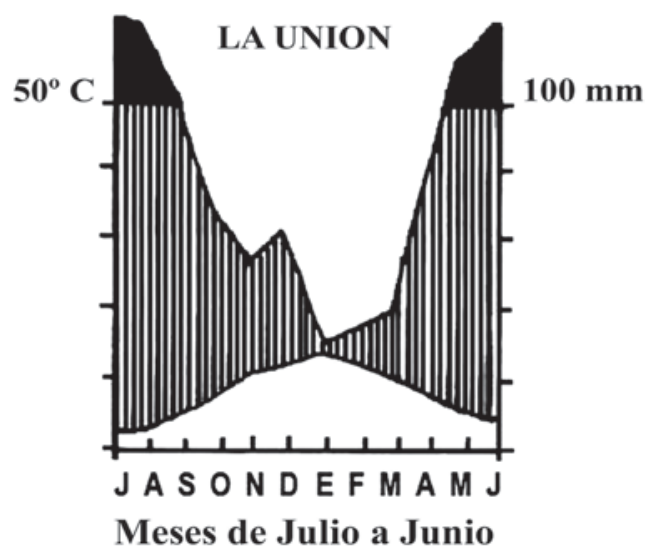

Figura 2. Diagrama climático de la ciudad de La Unión, Chile.

FIgURE 2. Climate diagram of La Union city, Chile.

De acuerdo a CIREN (2003) el suelo del lugar corresponde a la Serie Cudico, del tipo rojo arcilloso (Andetepic Palehumult), profundo, de color rojizo, con textura franco-arcillosa y con una alta densidad aparente (Mella \& Kühne 1985).

La vegetación potencial del lugar corresponde a un bosque de roble-laurel-lingue (Nothofago-Perseetum linguae Oberdorfer 1960), en su subasociación más xérica con boldo, denominada Nothofago-Peersetum linguae Boldetosum (Tomaselli 1981) y en cuyos estratos medios domina el boldo (Peumus boldus Molina), árbol de carácter esclerófilo (Ramírez et al. 2005). El paisaje vegetal actual está formado por restos intervenidos del bosque original, matorrales de zarzamora, praderas antropogénicas y tierras de cultivo, principalmente leguminosas y cereales (Golf 1973, San Martín et al. 1991).

\section{METODOLOGIA}

De la publicación de Ramírez et al. (2005) se tomaron las tablas fitosociológicas de vegetación representativas de cada manejo pratense descrito por ellos, a las que se agregó el valor indicador de cada especie para las variables luz (L) y temperatura $(\mathrm{T})$ del hábitat y reacción $(\mathrm{R})$, nitrógeno $(\mathrm{N})$ y humedad $(\mathrm{H})$ del suelo, tomados de Ramírez et al. (1991) y San Martín et al. (2003). Estos valores fluctúan de 1 a 9: el valor más alto indica una mayor y el más bajo una menor afinidad por el factor, siendo 5 el valor intermedio. El valor 0 (cero) indica indiferencia al factor, por ello no se considera en los cálculos para sacar los promedios. De estas tablas se obtuvieron valores promedios ponderados para cada factor de acuerdo a Ramírez et al. (1992) y, posteriormente, se procedió a la comparación de los manejos y de los estados de degradación, dentro de cada manejo. Con este propósito se distinguieron los siguientes estados: normal, alterado y muy alterado, considerando la desaparición de especies pratenses y la aparición de otras con escaso valor nutritivo. La metodología fitosociológica y la determinación de estados en los distintos manejos pratenses se describen en Ramírez et al. (2005).

La evaluación se realizó para las especies y para la cobertura de sus individuos, de manera que la última se interpreta como más adecuada ya que toma en cuenta la abundancia de cada una. Para ponderar la importancia de la cobertura de los individuos de cada especie se procedió a multiplicar cada factor indicador por la cobertura; luego se dividió la suma de los valores resultantes para cada variable por la cobertura total alcanzada por todos los censos de cada situación, según lo indicado por Ramírez et al. (1992) y Ramírez \& Finot (2006).

Para caracterizar la dinámica de degradación 
en los distintos manejos pratenses investigados, se realizaron análisis edáficos con las metodologías tradicionales de Sadzawka (1990) y Hartge \& Horn (1992). Los análisis se contrataron en el Instituto de Ingeniería Agraria y Suelos de la Facultad de Ciencias Agrarias de la Universidad Austral de Chile. Además, para estimar cambios en el suelo provocados por el manejo, se determinó “in situ” la resistencia a la penetración expresada en Kpa usando un penetrómetro de punta cónica (Forsythe 1980).

\section{RESULTADOS}

En las dos condiciones de manejo estudiadas, los valores indicadores con menor dispersión fueron los de luz y temperatura, es decir, los de condiciones microclimáticas del hábitat (Fig. 3). Los más altos valores de luz presentaron mayor número de especies, en cambio la temperatura tiende a concentrar las especies en los tramos medios de la escala. Por el contrario, los valores indicadores de características
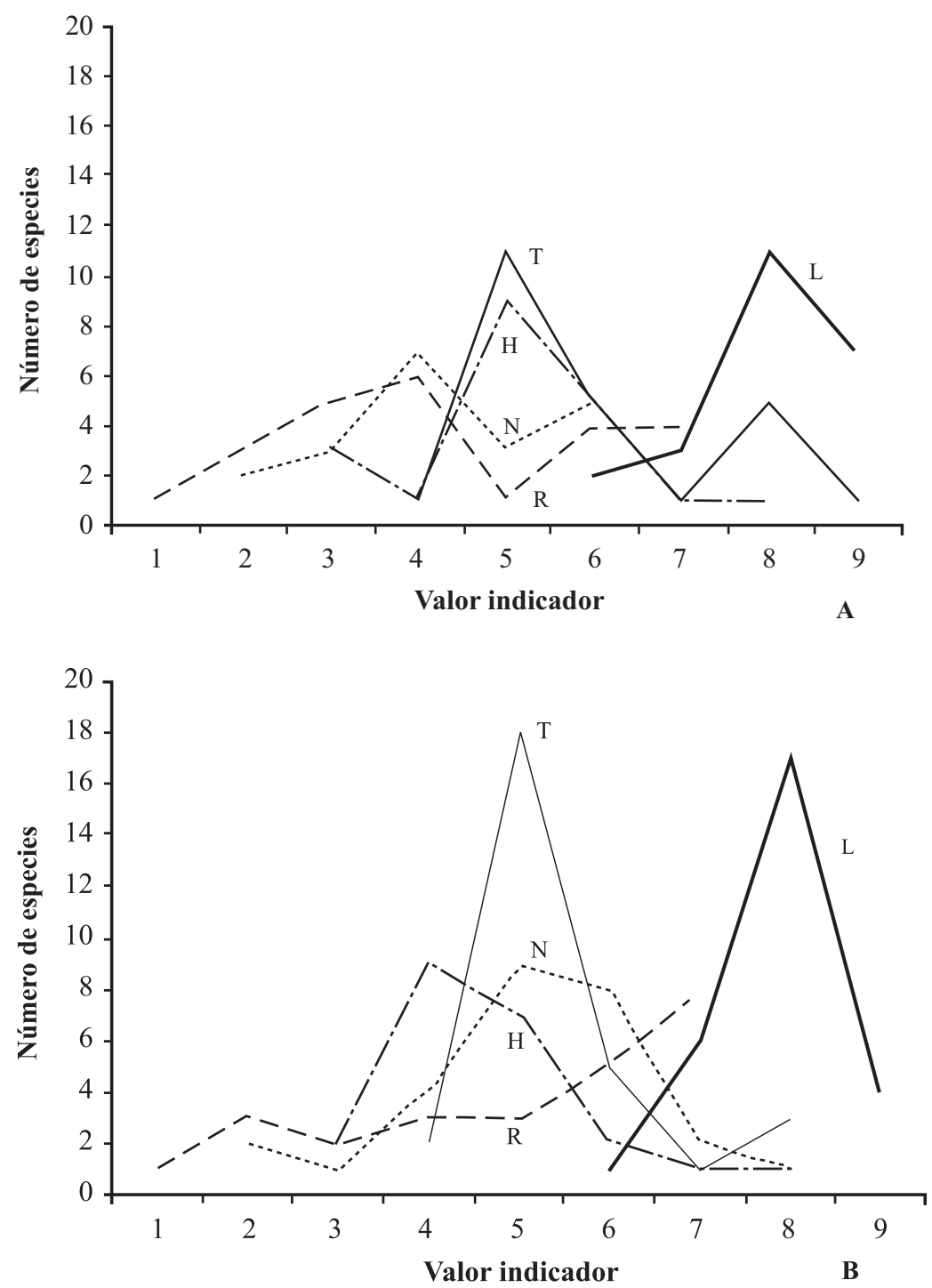

FIgURA 3. Valores indicadores ecológicos promedio por especies y cobertura para luz $(\mathrm{L})$ y temperatura $(\mathrm{T})$ y para reacción $(\mathrm{R})$, nitrógeno $(\mathrm{N})$ y humedad $(\mathrm{H})$ del suelo en la pradera de bovinos $(\mathrm{A})$ y de ovinos $(\mathrm{B})$.

Figure 3. Average ecological indicator values by species and cover for light (L), temperature (T), soil reaction (R), nitrogen $(\mathrm{N})$ and humidity $(\mathrm{H})$ of the studied cattle prairie $(\mathrm{A})$ and sheep prairie $(\mathrm{B})$. 
Gayana Bot. 66(2), 2009

edáficas, reacción, nitrógeno y humedad presentaron una mayor dispersión en la escala, incluso en algunos casos con una distribución bimodal. Para el nitrógeno, el mayor número de especies tiende a concentrarse en el tramo 4 en la pradera de ovinos y en los tramos 5 y 6 para la de bovinos. Es decir, hay una clara pérdida de nitrógeno en el manejo con ovinos de acuerdo a los indicadores biológicos utilizados. Para el indicador de reacción del suelo, en la pradera de bovinos se observa una tendencia a presentar un mayor número de especies en los valores altos, es decir, aquellos más básicos o con menor acidez. El indicador de humedad del suelo presentó resultados contrarios a lo esperado, ya que el mayor número de especies se concentró en el valor 4 en la pradera de vacunos y en el valor 5 en la de ovinos.
Pradera de Bovinos

Considerando el valor de indicador biológico de las especies, la pradera de bovinos presentó altos valores de luz (Tabla I), lo que está de acuerdo con la presencia de especies pratenses heliófilas. Los valores de afinidad hacia la temperatura, reacción del suelo, nitrógeno y humedad están próximos al valor medio. Al considerar la cobertura (abundancia) de los individuos de las especies indicadoras, todos los valores descienden con la única excepción de la humedad que aumenta levemente. El descenso es importante en el valor indicador promedio de reacción del suelo que llegó a 3,6, lo que señala condiciones de acidez, quizás debida a una disminución de nutrientes, típica de praderas permanentes no mejoradas.

TABLA I. Valores indicadores biológicos promedios para las praderas de bovinos y ovinos.

TABLE I. Average indicator biological values of the cattle and sheep prairies.

\begin{tabular}{cccccc}
\hline CÁlculos POR: & LuZ & TEMPERATURA & REAcción & NitróGeno & HumedAD \\
\hline & & & Pradera bovinos & & \\
Especies & 7,9 & 5,5 & 5,0 & 5,1 & 4,7 \\
Cobertura & 7,3 & 5,1 & 3,6 & 4,8 & 4,9 \\
Diferencia & $-0,6$ & $-0,4$ & $-1,4$ & $-0,3$ & 0,2 \\
& & & Pradera ovinos & & 4,2 \\
Especies & 8,0 & 6,0 & 4,4 & 4,6 & 3,5 \\
Cobertura & 7,9 & 6,4 & 3,5 & 4,1 & $-0,7$ \\
Diferencia & $-0,1$ & 0,4 & $-0,9$ & $-0,5$ & \\
\hline
\end{tabular}

Pradera de ovinos.

En esta pradera, los valores indicadores promedios de luz por especie alcanzan a ocho, lo que indica condiciones de alta luminosidad, propias de cualquier pradera (Tabla I). La temperatura muestra un valor relativamente alto, lo que señala condiciones de mayor insolación o menor cubierta vegetal. Los valores de reacción, nitrógeno y humedad del suelo se encuentran un poco por debajo de lo que se considera afinidad intermedia, lo que se asocia a una deficiencia de nutrientes, de materia orgánica y a mayores eventos de sequía. Al ponderar los valores de cobertura de las especies indicadoras, se aprecia en la mayoría de ellos un marcado descenso, con la única excepción del valor de temperatura que aumenta en un 0,4 , indicando condiciones más cálidas. La mayor disminución del valor indicador de humedad señala condiciones xéricas, seguramente por una compactación del suelo, que impide la retención del agua. El manejo pecuario altera la conductividad hidráulica en fase saturada de un suelo, la que decrece de acuerdo a la degradación del suelo (Ellies et al. 1997). El efecto del pisoteo animal y el tránsito de maquinaria reducen el espacio poroso del suelo, alterando la aireación y el movimiento y almacenamiento del agua (Seguel et al. 2002b, Dörner 2005, Dörner \& Horn 2006). 


\section{COMPARACIÓN DE PRADERAS}

POR ESPECIES

Al considerar solamente el número de especies indicadoras presentes en las praderas se observa un aumento de la luz, pero especialmente de la temperatura en la pradera de ovinos, indicando un mayor xerofitismo provocado por el pastoreo de ovejas, que compacta el suelo y disminuye la cubierta vegetal. Todos los valores indicadores de condiciones del suelo descienden en este mismo manejo en comparación con los valores del manejo con bovinos, señalando un deterioro de las características edáficas. Los mayores descensos indican una clara disminución en el contenido de nutrientes disponibles y de la materia orgánica, directamente asociado con un aumento de las condiciones de sequía del suelo. En general, se puede decir que todos los valores indicadores señalan una mayor degradación de la cubierta vegetal y del suelo con el pastoreo de ovinos en comparación con el de vacunos, lo que se relaciona no sólo con el pisoteo, sino también con el tamaño de la pata del animal que comprime el suelo con una fuerza determinada por unidad de superficie.

\section{POR COBERTURA}

Los valores indicadores que consideran la abundancia de los individuos de cada especie varían en la misma dirección que aquellos que consideran sólo el número de especies, pero las diferencias se hacen más marcadas en todas las características con la única excepción de la reacción del suelo. Efectivamente, la diferencia del indicador de Reacción es mayor considerando sólo las especies, lo que indica que en el manejo con bovinos hay una mayor cantidad de especies indicadoras de condiciones de acidez, mientras que en el manejo de bovinos disminuyen estas especies indicadoras, pero aumenta su abundancia. Al considerar la cobertura entonces, sólo se hace más evidente el deterioro de las condiciones edáficas y de la cubierta vegetal con un pastoreo de ovejas.

\section{COMPARACIÓN CON VALORES EDÁFICOS}

Las diferencias entregadas por los indicadores biológicos para las características del suelo de los dos manejos, coinciden plenamente con aquéllas obtenidas directamente de análisis edáficos físico-químicos. En la Tabla II se observa una

TABLA II. Valores de variables del suelo en las praderas de bovino y de ovinos.

TABLE II. Variables values of the soil in cattle and sheep prairies.

\begin{tabular}{cccc}
\hline VARiable edÁfica & Bovinos (CATtLe) & Ovinos (SheEP) & DiferenCia \\
\hline $\mathrm{pH}(\mathrm{en} \mathrm{Ca} \mathrm{Cl}$ ) & 4,7 & 4,5 & $-0,2$ \\
Materia orgánica $(\%)$ & 23,8 & 14,1 & $-19,7$ \\
Resistencia a la penetración $\left(\mathrm{kg} / \mathrm{cm}^{2}\right)$ & 39,93 & 44,96 & 5,03 \\
\hline
\end{tabular}

disminución del pH y de la materia orgánica del suelo en el pastoreo con ovinos en comparación con el de bovinos. En la resistencia a la penetración, en cambio, se obtuvo un mayor valor en el suelo sometido al pastoreo con ovinos, lo que también señala un aumento del xerofitismo y una pérdida de la humedad del suelo, como también lo confirman los valores dados por los indicadores biológicos. $\mathrm{La}$ resistencia a la compactación es una medida de la degradación física del suelo, que pierde estructura y, por lo tanto, altera su conductividad hidráulica. Esta comparación permite validar las evaluaciones hechas con los indicadores biológicos en praderas permanentes del centro-sur de Chile (Ramírez et al. 2003b).

\section{ESPECIES INDIFERENTES}

El número de especies indiferentes y, por lo tanto, sin valor indicador biológico hacia un determinado factor fue similar en ambos manejos para luz, temperatura y nitrógeno, dentro de los cuales este último presentó el mayor número de especies indiferentes (Fig. 4). Para los factores de reacción y humedad del suelo en cambio se presentó un 
mayor número de especies indiferentes en el manejo con bovinos. Esto indica que las especies indiferentes comienzan a desaparecer cuando las condiciones se hacen más extremas y aumenta la competencia, como es el caso de la pradera de ovinos. Aunque las especies indiferentes pueden serlo por varios factores, se puede concluir que en la pradera de vacunos hay una mayor cantidad de especies indiferentes como indicadores biológicos, lo que señala una mejor condición del biótopo en ella que en la pradera mantenida con ovejas.

ESTADOS DE DEGRADACIÓN

MANEJO CON BOVINOS

Al considerar el número de especies por indicador biológico, en los estados de degradación de la pradera mantenida con bovinos se observa que las diferencias en los valores entre los distintos estados de degradación son poco marcadas (Tabla III). Se ve efectivamente un descenso en la mayoría de los factores, aunque pequeño. La única excepción la constituye el factor humedad del suelo, que curiosamente aumenta en la pradera degradada y $\sin$ embargo, en la muy degradada vuelve a recuperar el valor original. Sólo la temperatura muestra un descenso constante hacia la pradera muy degradada, pero como se comentó antes las diferencias son mínimas. Al parecer en el estado muy degradado, por la ausencia de animales, el biótopo comienza a presentar etapas propias de la sucesión secundaria.
Al considerar la cobertura de las especies indicadoras, se observa un comportamiento muy similar al observado con el número de especies, es decir, también hay diferencias pequeñas y sin una tendencia muy clara. En todo caso, los valores y sus tendencias son comparables con los de las especies, aunque en este último caso la reacción y el nitrógeno del suelo muestran valores más bajos, indicando un fuerte deterioro del biótopo.

\section{MANEJo CON OVINOS \\ El comportamiento de los valores indicadores} biológicos entregados por el número de las especies en los diferentes estadios de degradación del manejo con ovinos no muestra gran diferencia. Sólo en los factores reacción y nitrógeno del suelo se presenta una leve tendencia a la baja, la que se torna muy marcada para la reacción del suelo en la pradera muy degradada, lo que acusa una importante pérdida de nutrientes con este manejo. Al considerar la cobertura de las especies indicadoras, las diferencias entre los estados de degradación son similares a aquéllas obtenidas con el número de especies (Tabla IV). En este caso, sólo se observa un claro deterioro en la reacción del suelo, confirmando una pérdida de nutrientes con el consiguiente empobrecimiento del suelo. Estos valores muestran entonces, una marcada tendencia a la acidez y a la pérdida de materia orgánica con el pastoreo de ovejas, confirmado por la disminución de los parámetros de suelo en el análisis de abundancia con respecto al número de especies.

TABLA III. Valores indicadores biológicos promedios para distintos estados de degradación de la pradera de bovino, considerando número y abundancia de especies.

TABLE III. Average biological indicators values of different status of the cattle prairie degradation, considering number and abundance of species.

\begin{tabular}{cccccc}
\hline Manejo & Luz & Temperatura & Reacción & NitróGeno & Humedad \\
\hline Normal & 7,9 & 5,5 & Número de especies & 5,1 & 4,7 \\
Degradada & 7,8 & 5,4 & 5,0 & 5,0 & 5,1 \\
Muy degradada & 7,8 & 5,3 & 4,6 & 4,9 & 4,7 \\
& & & Abundancia de especies & & 4,9 \\
Normal & 7,3 & 5,1 & 3,6 & 4,8 & 5,2 \\
Degradada & 7,1 & 5,1 & 3,2 & 3,2 & 4,9 \\
Muy degrada & 7,2 & 5,1 & 3,2 & 4,6 & \\
\hline
\end{tabular}


Bioindicadores pratenses de Ellenberg: SAN MARTín, C. ET AL.

TABLA IV. Valores indicadores biológicos promedios para distintos estados de degradación de la pradera de ovino, considerando número y abundancia de especies.

TABLE IV. Average biological indicators values of different status of the sheep prairie degradation, considering number and abundance of species.

\begin{tabular}{cccccc}
\hline MANejo & LuZ & Temperatura & Reacción & Nitrógeno & HumedAD \\
\hline Normal & 8,0 & 6,0 & Número de especies & 4,6 & 4,2 \\
Degradada & 8,0 & 6,2 & 4,4 & 4,5 & 3,9 \\
Muy degradada & 7,9 & 5,9 & 3,6 & 4,3 & 4,4 \\
& & \multicolumn{5}{c}{ Abundancia de especies } \\
Normal & 7,9 & 6,4 & 3,5 & 4,1 & 3,5 \\
Degradada & 8,0 & 6,6 & 3,5 & 4,1 & 3,2 \\
Muy degradada & 7,6 & 6,1 & 2,5 & 3,9 & 3,9 \\
\hline
\end{tabular}

PoR ESPECIES

Al comparar los estados de degradación de ambos manejos pratenses (pradera de ovinos menos pradera de bovinos) calculando los valores indicadores de las especies (Fig. 5A), los factores del hábitat (luz $\mathrm{y}$ temperatura) presentan un aumento en los tres estados, sin embargo, sólo en el factor temperatura muestran diferencias considerables, lo que indica que con el manejo de ovinos hay un aumento de las condiciones térmicas del biótopo. Los factores reacción, nitrógeno y humedad del suelo muestran menores valores en todos los estados de degradación del manejo de ovinos, en comparación con el manejo de bovinos. Las diferencias son claras, pero también algo erráticas, ya que no siempre los mayores descensos aparecen en el estadio más degradado. Valores bajos destacables se presentan en la reacción del suelo en la pradera muy degradada y de humedad, en la degradada.

POR COBERTURA

Al considerar la cobertura (Fig. 5B), las diferencias entre manejos (ovino menos bovino) para los distintos estados de degradación conservan las tendencias observadas con las especies, pero se hacen mayores. De los dos factores del hábitat la temperatura muestra diferencias importantes, alcanzando valores sobre 1. Esto indica que en el manejo de ovinos las características xéricas del hábitat se acentúan. Las diferencias en los factores edáficos de reacción, nitrógeno y humedad son negativas, indicando una degradación del biótopo con manejo de ovinos. Sólo en la pradera degradada, la reacción y el nitrógeno del suelo presentan diferencias positivas, lo que indica que en los primeros estados de degradación estarían aumentando estos dos factores, seguramente por la acción del ganado que aporta materia orgánica, con nutrientes, incrementando la eutrofización del suelo. Los valores presentados en la Fig. 5 hablan de un aumento en la sequía edáfica en el manejo con ovino, lo que se debe a la acción de pisoteo, que compacta el suelo, disminuyendo la porosidad y cambiando su distribución.

\section{MATORRAL DE ZARZAMORA}

En la pradera manejada con pastoreo de bovinos se encontró un estadio con Rubus constrictus P.J.Müll. et Lefèvre (zarzamora) que al parecer coloniza por abandono del pastoreo y un biótopo que aún presenta claros indicios de degradación, como lo señalan los valores indicadores calculados. Al considerar el número de especies (Tabla V) se observan valores bajos de temperatura, causados por el sombreamiento del dosel de zarzamora. Todas las características del suelo se presentan con valores bajo el intermedio (5). Al considerar la cobertura de las especies indicadoras, se presenta una disminución de los valores de luz, temperatura y reacción del suelo, pero un aumento del nitrógeno y de la humedad del suelo, confirmando el efecto sombreador y de aporte de materia orgánica (follaje) de la zarzamora que es caducifolia. 
Al comparar el sitio de zarzamora con los distintos estados de la pradera (Tabla V), se obtienen valores iguales para la luz y la humedad del suelo, pero menores para la temperatura del hábitat y la reacción y el nitrógeno del suelo. Los descensos son bastante marcados en los factores del suelo, lo que confirma el hecho de que la zarzamora ha invadido un hábitat previamente degradado.
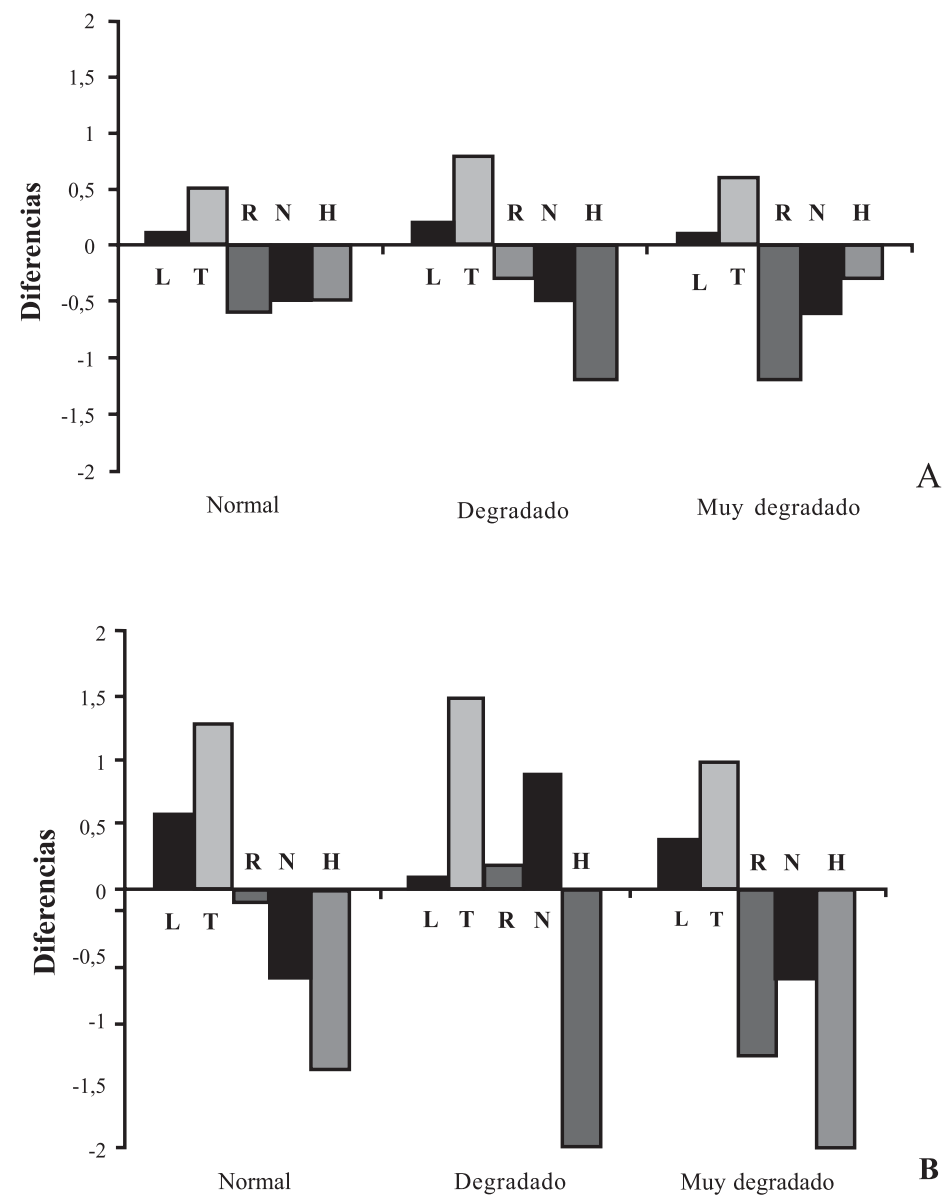

FIGURA 5. Diferencias positivas y negativas (ovinos menos bovinos) entre los valores indicadores promedio (A) número de especies y (B) cobertura. El valor de bovinos se consideró cero.

Figure 5. Positive and negative differences between (sheep minus cattle) the average indicator values (A) number of species and (B) cobertura. The cattle management are considered zero.

TABLA V. Valores indicadores promedio por especies y cobertura del estado degradado con zarzamora (matorral de zarzamora) de la pradera de bovinos.

TABLE V. Average indicator values for species and cover of the degradation status of zarzamora (zarzamora busch) of the cattle prairie.

\begin{tabular}{cccccc}
\hline CÁlculos POR: & LuZ & TEMPERATURA & REACCIÓN & NitrÓGeNO & HumEDAD \\
\hline Especies & 7,8 & 5,2 & 4,2 & 4,3 & 4,7 \\
Cobertura & 7,3 & 5,1 & 3,8 & 5,3 & 4,8 \\
Diferencia & $-0,5$ & $-0,1$ & $-0,4$ & 1,0 & 0,1 \\
\hline
\end{tabular}


Considerando la abundancia de individuos las tendencias cambian claramente, ya que en luz y temperatura no hay mayores diferencias, pero los valores de la reacción y del contenido de nitrógeno del suelo aumentan en forma considerable, especialmente en el último; esto tiene la misma explicación dada anteriormente en el sentido del gran aporte de materia orgánica de la zarzamora caducifolia al suelo. Se observa también una leve disminución de la humedad en el matorral de zarzamora, lo que seguramente se debe a la tardanza en recuperar la estructura física del suelo, que ha perdido su porosidad.
En las dos series de degradación (bovino y ovino) se aprecia un claro deterioro en el número de especies vegetales presentes y también en la cobertura (Fig. 6). Por lo anterior, habría que pensar que los pequeños cambios en las características del biótopo influyen fuertemente en la composición florística y en la cobertura vegetal de ambos manejos. La pobreza de especies en el matorral de zarzamora indica quizás la degradación del biótopo, que homogeneiza sus condiciones, pero también podría ser producto del sombreado.

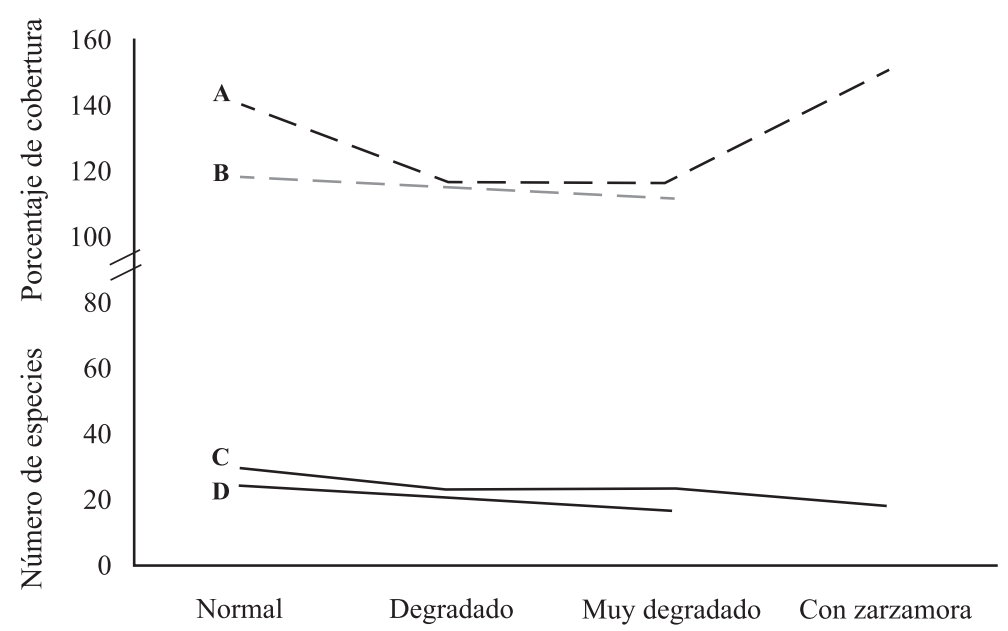

Figura 6. Número de especies (C y D) y porcentaje de cobertura (A y B) del manejo de bovinos (A y C) y de ovinos (B y D).

FigurE 6. Number of species (C and D) and cover percentage (A and B) of the cattle management (A and C) and of the sheep management (B and D).

\section{DISCUSION}

Los indicadores biológicos de luz y temperatura son más bien dependientes del microclima del lugar, mientras que los de reacción, nitrógeno y humedad se pueden considerar edáficos. Los primeros concentran en pocos tramos las especies indicadoras, mientras que los segundos presentan una mayor dispersión en la escala de Ellenberg (1979). Al considerar sólo el número de especies indicadoras ya es posible observar diferencias claras entre los manejos, las que se hacen aún más nítidas al considerar la cobertura (Ramírez et al. 1992). Por ello, permiten evaluar y comparar las condiciones de sitio, creadas por los distintos manejos (cambios antropogénicos) que cambian la composición florística y las condiciones del hábitat.

Todos los bioindicadores utilizados en este estudio señalan que el pastoreo con ovinos degrada el suelo y la cubierta vegetal, mucho más que el pastoreo con bovinos, lo que coincide con la degradación florística y del espectro biológico en ambos manejos pratenses (Ramírez et al. 2005). Se confirma que es muy importante en el manejo pastoril la degradación física del suelo, especialmente por la compactación que impide la penetración y la retención del agua en el suelo. En 
su degradación física, el suelo pierde estructura (porosidad y dirección de los poros) y por ello se altera su conductividad hidráulica, disminuyendo la porosidad y aumentando la sequía (Ellies et al. 1993). Así se obtiene un mayor xerofitismo antrópico que trae consigo una disminución de la cubierta vegetal. La compactación del suelo dificulta además el arraigamiento, favoreciendo el desarrollo de hierbas anuales con sistemas radicales profusos, pero superficiales en el manejo pratense con ovinos (Ramírez et al. 2005). Por el contrario, en la pradera de bovinos menos degradada, dominan hierbas perennes, con un arraigamiento más profundo (Ramírez et al. 1996, Ellies 1995).

De acuerdo a los resultados, se observa un claro deterioro físico y químico de las condiciones edáficas con el manejo de ovinos: compactación y un aumento de la tortuosidad del sistema poroso, pérdida de nutrientes y especialmente de materia orgánica, que provoca una fuerte acidificación del suelo. La degradación vegetacional sigue cursos diferentes en los distintos tipos de pastoreo como lo comprobaran Ramírez et al. (2005).

Al parecer, en estados muy degradados por la ausencia del pastoreo, el biótopo comienza a recuperar sus características primitivas, con la llegada de especies nativas que cambiarán el curso de la sucesión. Por el contrario, en los primeros estados de intervención pastoril y con cierto manejo de la cubierta pratense, se logra una rápida eutrofización del suelo, especialmente al incorporar purines. Las variaciones de los estados de degradación muestran que hay un deterioro gradual de los ambientes, en la cual cada uno de los estados podría considerarse como un biótopo diferente (Ramírez \& Finot 2006).

Pocas especies indiferentes para un determinado factor indican que dicho factor ambiental afecta a todas las especies, mientras que muchas especies indiferentes a un factor pueden indicar que él no es muy decisivo en la distribución de las especies, ya que no las afecta. La mayor cantidad de especies indiferentes a condiciones de sitio predice condiciones favorables. Aunque también se ha demostrado una relación directa entre el número total de especies y la presencia de especies indiferentes. Efectivamente, a mayor cantidad de especies aparece un mayor número de especies indiferentes, sin embargo, en condiciones más extremas, esta correlación desaparece (Ramírez et al. 2000).
El matorral de zarzamora ayuda a la recuperación del suelo degradado, al otorgar sombreamiento que excluye las malezas intolerantes; además, aporta materia orgánica con la caducidad de sus hojas. Este matorral correspondería a los primeros estados de la sucesión hacia un matorral de maqui y zarzamora (Aristotelio-Rubetum constrictae Hildebrand 1983) muy frecuente en ambientes ruderales de tierras bajas del centro-sur de Chile.

La base de la utilidad de los indicadores biológicos vegetales reside en los comportamientos fisiológico y ecológico. El primero consiste en aquel comportamiento de la planta en un cultivo puro sin competencia, el segundo es el comportamiento en cultivo mixto, es decir, con la competencia de al menos otra especie. Este último comportamiento es el que presentan todas las plantas en sus hábitats naturales (o artificiales, pero en el terreno). En cultivo puro, una especie puede crecer aun en condiciones desfavorables, pero en cultivo mixto sólo puede hacerlo en condiciones óptimas para ella, es decir, los requerimientos en este caso se ven restringidos por la competencia (Dierschcke 1994), por lo que cada especie es relegada a un biótopo, del cual pasa a ser indicadora (Ramírez et al. 1995). Las especies y valores indicadores son útiles para caracterizar un hábitat y pueden ser usados, sin grandes costos ni mediciones prolongadas, por cualquier persona, siempre que se someta a un entrenamiento previo (Hawkes et al. 1997).

Los resultados de este estudio se corresponden con las evaluaciones realizadas en otras praderas permanentes del centro-sur de Chile, confirmando que ellos sirven para hacer en forma rápida $\mathrm{y}$ barata la evaluación del estado del suelo después de un manejo agropecuario prolongado (Ramírez et al. 2000). Los valores indicadores creados en Europa tienen una gran aplicación y utilidad en las praderas del sur de Chile, debido a que en ellas son dominantes especies de malezas europeas, que por su cuerpo herbáceo son más plásticas para colonizar ambientes ruderales sometidos a la permanente acción humana (Ramírez \& Finot 2006).

Pequeños cambios en las características del biótopo influyen fuertemente en la composición florística y en la cobertura vegetal (Ramírez et al. 1997). El ganado ayuda a mantener la estabilidad de los estados pratenses más eutrofizados, siempre y cuando no se provoque una degradación excesiva que destruya la cubierta vegetal (Hickman et al. 2004). Esto exige un manejo de dichos ecosistemas 
seminaturales. Por ello en exclusiones se provoca un brusco empobrecimiento de la flora, disminuyendo las especies anuales y la compactación, el pH y el contenido de nutrientes (Ramírez et al. 1997).

Aunque la vegetación boscosa original está determinada por factores climáticos tan importantes como la humedad y la temperatura (Ramírez et al. 1984, Ramírez \& Figueroa 1985) en la degradación, en los manejos pratenses pasan a tener importancia factores que tienen que ver con la degradación física y química del suelo (Ramírez et al. 1984). Sin embargo, la exclusión de ganado suele provocar un empobrecimiento de la flora y la pérdida de nutrientes del suelo.

\section{AGRADECIMIENTOS}

Se agradece el apoyo financiero de la Dirección de Investigación y Desarrollo de la Universidad Austral de Chile, mediante el Proyecto DID-UACh $\mathrm{N}^{\circ} \mathrm{S}-$ 2000-25. A los revisores anónimos por aportarnos sus valiosos comentarios y sugerencias.

\section{BIBLIOGRAFIA}

Amigo, J. \& C. RAmírez. 1998. Abioclimatic classification of Chile: woodland communities in the temperate zone. Plant Ecology 136: 9-26.

CIREN. 2003. Estudio Agroecológico X Región. Publicación del Centro de Investigaciones en Recursos Naturales (CIREN, CORFO) (1) 123: 1-199.

Di Castri, F. \& E. Hajek. 1976. Bioclimatología de Chile. Universidad Católica de Chile, Santiago $128 \mathrm{pp}$.

DieKMann, M. 1995. Use and improvement of Ellenberg's indicator values in deciduous forest of the Boreo-nemoral zone in Sweden. Ecography 18: 178-189.

DierschCKe, H. 1994. Pflanzensoziologie - Grundlagen und Methoden. Eugen Ulmer, Stuttgart. 683 pp.

DöRNER, J.M. 2005. Anisotropie von Bodenstrukturen und Porenfunktionen in Böden und deren Auswirkungen auf Transportprozesse im gesättigten und ungesättigten Zustand. $\mathrm{Ph}$. D. Thesis. Schriftenreihe des Instituts für Pflanzenernährung und Bodenkunde, Christian Albrechts University, Kiel, Germany. 182 pp.

Dörner, J.M. \& R. Horn. 2006. Anisotropy of pore functions in structured stagnic luvisols in the weichselian moraine region in Northern Germany. Journal of Plant Nutrition and Soil Science 169: 213-220.

EllenberG, H. 1979. Zeigerwerte der Gefässpflanzen
Mitteleuropas. 2. Aufl. Scripta Geobotanica 9: 5-97.

Ellenberg, H., H. Weber, R. Düll, V. Wirth, W. Werner \& D. Paulissen. 1992. Zeigerwerte von Pflanzen in Mitteleuropas. Scripa Geobotanica 18: 5-258.

ELLIES, A. 1995. Efecto del manejo sobre las propiedades físicas de suelos trumaos y rojo arcillosos. Bosque 16(2): 101-110.

Ellies, A., R. Grez \& C. Ramírez. 1997. La conductividad hidráulica en fase saturada como herramienta para el diagnóstico de la estructura del suelo. Agro Sur 25(1): 51-56.

Ellies, A., C. Ramírez, \& R. MacDonald. 1993. Cambios en la porosidad de un suelo por efecto de su uso. Turrialba 43(1): 77-82.

Ertsen, A.C.D., J.R.M. Alkemade \& M.J. Wassen. 1998. Calibrating Ellenberg indicator values for moisture, acidity, nutrient availability and salinity in the Netherlands. 135: 113-124.

Forsythe, W. 1980. Física de suelos. Manual de laboratorio. Instituto Interamericano de Ciencias Agrícolas (IICA), San José de Costa Rica. 212 pp.

Godefroid, S. \& E. Dana. 2007. Can Ellenberg's indicator values for Mediterranean plants be used outside their region of definition? Journal of Biogeography 34: 62-68.

GoLf, E. 1973. Über gegenseitige Beeinflussungen und Nährstoffwirkungen bei Arten der anthropogenen RasenvegetationvonsüdlichenMittel-Chilewährend der Keimung und des Wachstum. Dissertation, Justus Liebig Universität, Giessen. 230 pp.

Hartge, K.H. \& R. Horn. 1992. Die physikalische Untersuchung von Böden. Ferdinand Enke Verlag, 3. Auflage, Stuttgart, 177 pp.

Hawkes, J.C., D.G. PyatT \& I.M.S. Whitw. 1997. Using Ellenberg indicator values to asses soil quality in British forest from ground vegetation: a pilot study. Journal of Applied Ecology 34: 375-387.

Hickman, K., D.C. Harnett, R. Cochran \& C.E. OwensBy. 2004. Grazing management effects on plant species diversity in all grass prairie. Rangeland Ecology \& Management 57(1): 58-65.

Hildebrand, R. 1983. Die Vegetation der Tiefslandgebüsche des südchilenischen Loorbeerwaldgebiete uner besonderer Berücksichtigung der Neophyten Problematik. Phytocoenologia 11(2): 145-223.

Landolt, E. 1977. Oekologische Zeigerwerte zur Schweizer flora. Ver. Geobot. Inst. ETH, Stifftung Rübel Zürich 64: 5-208.

Mella, A. \& A. KüHNE. 1985. Sistemática y descripción de las Familias, Asociaciones y Series de suelos derivados de materiales piroclásticos de la Zona Central-Sur de Chile. En: J. Tosso (ed.), Suelos volcánicos de Chile. INIA, Santiago, Chile. pp: 548-716.

NovoA, R. \& S. Villaseca. 1989. Mapa agroclimático de Chile. Instituto de Investigaciones Agropecuarias (INIA), Santiago. $221 \mathrm{pp}$. 
Gayana Bot. 66(2), 2009

OBERDORFER, E. 1960. Pflanzensoziologischen Studien in Chile - Ein Vergleich mit Europa. Flora et Vegetatio Mundi 2: 1-208.

Ramírez, C. \& H. Figueroa. 1985. Delimitación ecosociológica del Bosque Valdiviano (Chile) mediante análisis estadístico multivariados. Studia Oecologica 5: 105-124.

Ramírez, C. \& V. Finot. 2006. Ruderal flora from the city of Valdivia (Chile) and their ecological indicator-value spectra. Revista Geográfica de Valparaíso 38: 69-80.

Ramírez, C., J. Amigo \& C. SAn Martín. 2003b. Vegetación pratense litoral y dinámica vegetal antropogénica en Valdivia, Chile. Agro Sur 31(1): 24-37.

Ramírez, C., M. Moraga \& H. Figueroa. 1984. La similitud florística como medida de degradación antrópica del bosque valdiviano. Agro Sur 12(2): 127-139.

Ramírez, C., C. San Martín \& C. Grüner. 2005. Cambios florísticos y vegetacionales con diferentes manejos pecuarios en un suelo Andeptic Palehumults (La Unión, X Región, Chile). Agro Sur 33(2): 13-28.

Ramírez, C., C. San Martín \& M.L. Keim. 2000. Evaluación ecológica del antiguo basural de Ovejería (Osorno, Chile). Agro Sur 28(2): 77-86.

Ramírez, C., A. Ellies, R. MacDonald \& O. Seguel. 2003a. Cambios en la flora nativa y la materia orgánica desde bosques nativos a praderas antropogénicas en suelos volcánicos de la IX Región de Chile. Revista de la Ciencia del Suelo y Nutrición Vegetal 2(2): 1-12.

Ramírez, C., A. Ellies, G. Torres \& C. San Martín. 1996. Degradación de la cubierta vegetal y su efecto sobre el arraigamiento. Boletín de la Sociedad Chilena de la Ciencia del Suelo 11: 10-18.

Ramírez, C., V. Finot, C. San Martín \& A. Ellies. 1991. El valor indicador ecológico de las malezas del Centro-Sur de Chile. Agro Sur 19 (2): 94-116.

Ramírez, C., C. San Martín, A. Ellies \& R. MacDonald. 1997. Cambios florísticos, fitosociológicos y edáficos provocados por exclusión de pastoreo en una pradera valdiviana, Chile. Agro Sur 24(2): 180-195.

Ramírez, C., C. San Martín, V. Finot \& A. Ellies. 1995. Diferenciación de manejos agropecuarios en un suelo trumao (andisol) usando indicadores ecológicos. Ciencia e Investigación Agraria 22 $(1-2): 3-14$

Ramírez, C., C. San Martín, V. Finot \& D. Ríos. 1992. Evaluación de praderas usando indicadores ecológicos. Agro Sur 20 (2): 85-100.

SadzawKa, A. 1990. Métodos de análisis de suelos. Instituto de Investigaciones Agropecuarias (INIA), Serie La Platina 16: 1-130.

San Martín, C., C. Ramírez \& M. Alvarez. 2003. Macrófitos como bioindicadores: una propuesta metodológica para caracterizar ambientes dulciacuícolas. Revista Geográfica de Valparaíso 34: 243-253.

San Martín, C., G. Rodríguez \& C. Ramírez. 1991. Origen de la vegetación actual del sector RapacoPichirropulli (Valdivia, Chile). Actas II Congreso Internacional de Gestión en Recursos Naturales (Valdivia) 2: 456-468.

Schaffers, A. \& K. SyKOra. 2000. Reliability of Ellenberg values for moisture, nitrogen and soil reaction; a comparison with field measurements. Journal of Vegetation Science 11: 225-244.

Scherhag, R. 1969. Klimatologie. Das Geographisches Seminar, Westermann Verlag, Braunschweig. $165 \mathrm{pp}$

Seguel, O., A. Ellies, R. MacDonald \& C. Ramírez. 2002a. Capacidad de soporte y resistencia al corte en suelos sometidos a distinto uso. Boletín de la Sociedad Chilena de la Ciencia del Suelo 18: 47-50.

Seguel, O., A. Ellies, R. MacDonald \& C. Ramírez. 2002b. Propiedades mecánicas en suelos sometidos a distintos usos. Revista de la Ciencia del Suelo y nutrición vegetal 2 (2): 56-61.

TomaselLI, R. 1981. The longitudinal zoning of vegetation in the southern sector of the Andes. Studi Trentini di Scienze Naturali, Acta Biologica 58: 471-484. 